\title{
DELIMITACIÓN Y CARACTERIZACIÓN DE UNIDADES GEOLÓGICAS A PARTIR DEL MODELADO NUMÉRICO DEL TERRENO EN EL DISTRITO DE JUAN VIÑAS, CARTAGO
}

\author{
SETTING AND CHARACTERIZATION OF GEOLOGICAL \\ UNITS FROM LAND NUMERICAL MODEL IN THE \\ DISTRICT OF JUAN VIÑAS, CARTAGO
}

\author{
Héctor Zúñiga Mora ${ }^{1}$ \\ Pablo Ramírez Granados ${ }^{2}$ \\ Universidad Nacional, Costa Rica
}

\section{RESUMEN}

Se delimitan las unidades geológicas del distrito de Juan Viñas a partir del uso de modelado numérico del terreno como una forma de zonificar el área de estudio. Dicho modelado implicó el uso de curvas de nivel vectoriales para la creación del MED el cual sirvió como base para el modelo de sombras sobre el cual se delinearon las unidades Adicionalmente se realizaron ensayos, recolección de muestras macroscópicas para descripción básica y para sección delgada, para establecer la naturaleza petrográfica de los materiales litológicos encontrados. Fueron propuestas diez unidades geológicas asociadas principalmente a coladas lávicas de origen diverso, identificándose flujos generados a partir de un único punto de emisión, emisiones lineales a través de fisuras o domos de forma subcónicas. Estas unidades son cubiertas por depósitos superficiales que podrían corresponder

1 Investigador, Centro de Investigaciones en Ciencias Geológicas (CICG), Universidad de Costa Rica, enrique.zuniga@ucr.ac.cr.

2 Investigador, Laboratorio de Hidrogeología y Manejo de Recursos Hídricos (LHMRH), Escuela de Ciencias Ambientales (EDECA), Universidad de Costa Rica, pablo.ramirez.granados@una.cr 
Héctor Zúñiga Mora, Pablo Ramírez Granados. Setting and characterization of geological units from land numerical model in the district of Juan Viñas, Cartago

con depósitos de ceniza meteorizados. También han sido identificadas unidades de valles y terrazas aluviales, escarpes de posible origen tectónico y depósitos de flujos en masa.

Palabras clave: Unidades geológicas, modelado numérico del terreno, MED, Juan Viñas

\begin{abstract}
Geological units of the district of Juan Viñas were established based on the use of a land numerical modeling as a form of zoning the studying area. Such modeling involved the use of vector contour lines to create the MED which served as the basis for the shaded relief map where the geological units were delimited. Additionally, some tests and sample collection for the basic macroscopic description and thin sections were performed in order to establish the petrographic nature of the lithological materials that were found. Ten geological units mainly related to lava flows, thus identifying flows generated from a single point of emission, lineal emissions through cracks or subconical domes were suggested. These units were covered with surface deposits that may correspond to wheathered ash deposits. Valley units and alluvial terraces, possible tectonic scarps and massive flow deposits have been also identified.
\end{abstract}

Keywords: Geological units, land numerical modeling, DEM, Juan Viñas.

\title{
Introducción
}

En algunos lugares en Costa Rica (si bien en la actualidad existe un amplio conocimiento de las unidades geológicas que conforman el paisaje) existen aún áreas donde la información es escasa e incluso nula. Esta escasez muchas veces va de la mano con diversas situaciones: la ausencia, parcial o total, de estudios geológicos regionales de escala menor a 1:50.000, que fundamenten o justifiquen la necesidad de elaborar un estudio geológico local, la falta de un estudio geológico detallado que incluya un robusto análisis de campo y la ausencia de descripciones litológicas de pozos que faciliten el reconocimiento de los materiales en superficie y en profundidad. Si bien existen algunos mapas geológicos regionales, como el mapa de Denyer \& Alvarado (2007), estos presentan una escala que imposibilita su uso para trabajos más detallados. Para la zona de estudio, el trabajo de Fernández (1987), es el que presenta una mayor cercanía, sin embargo sigue siendo muy regional pues se establece en toda la hoja Tucurrique.

En el caso del distrito de Juan Viñas, la recuperación bibliográfica solo identificó un trabajo específico en la zona, relacionado a un estudio geotécnico de un sector (Mora, 2000) lo cual no es suficiente para tener una guía local sobre la estructura geológica del área de estudio. Debido a esto, se generó una extensa campaña de trabajo de campo, ensayos y 
trabajo de laboratorio, para generar un primer estudio geológico de la zona de estudio que pueda servir como base para la elaboración futura de otros estudios más detallados y aplicados. Este trabajo es introductorio al entendimiento de las unidades geológicas locales que conforman el distrito de Juan Viñas, como todo trabajo inicial, este puede ser ampliado y mejorado en el futuro.

Este artículo surge como un producto parcial de investigación científica del proyecto 830-B2-039 denominado "Estudio hidrogeológico en el distrito de Juan Viñas, Cartago” (Ramírez \& Zúñiga, 2014) inscrito en la Vicerrectoría de Investigación de la Universidad de Costa Rica. Dicho estudio surgió a partir de una solicitud de colaboración de parte de la Municipalidad de Jiménez al Centro de Investigaciones en Ciencias Geológicas, el cual generó un convenio de colaboración y donde los autores colaboraron como el personal científico encargado de la generación de la información.

\section{Área de Estudio}

El distrito de Juan Viñas, cabecera del cantón Jiménez de la provincia de Cartago, se ubica dentro de las coordenadas 560.453 y $568.393 \mathrm{~m}$ este y 204.426 y 214.752 m norte del sistema de proyección Conforme Cónico de Lambert para Costa Rica Norte (Figura 1), presenta una extensión de 43,62km². 
Héctor Zúniga Mora, Pablo Ramírez Granados. Setting and characterization of geological units from land numerical model in the district of Juan Viñas, Cartago

Figura 1. Mapa de ubicación del área de estudio.

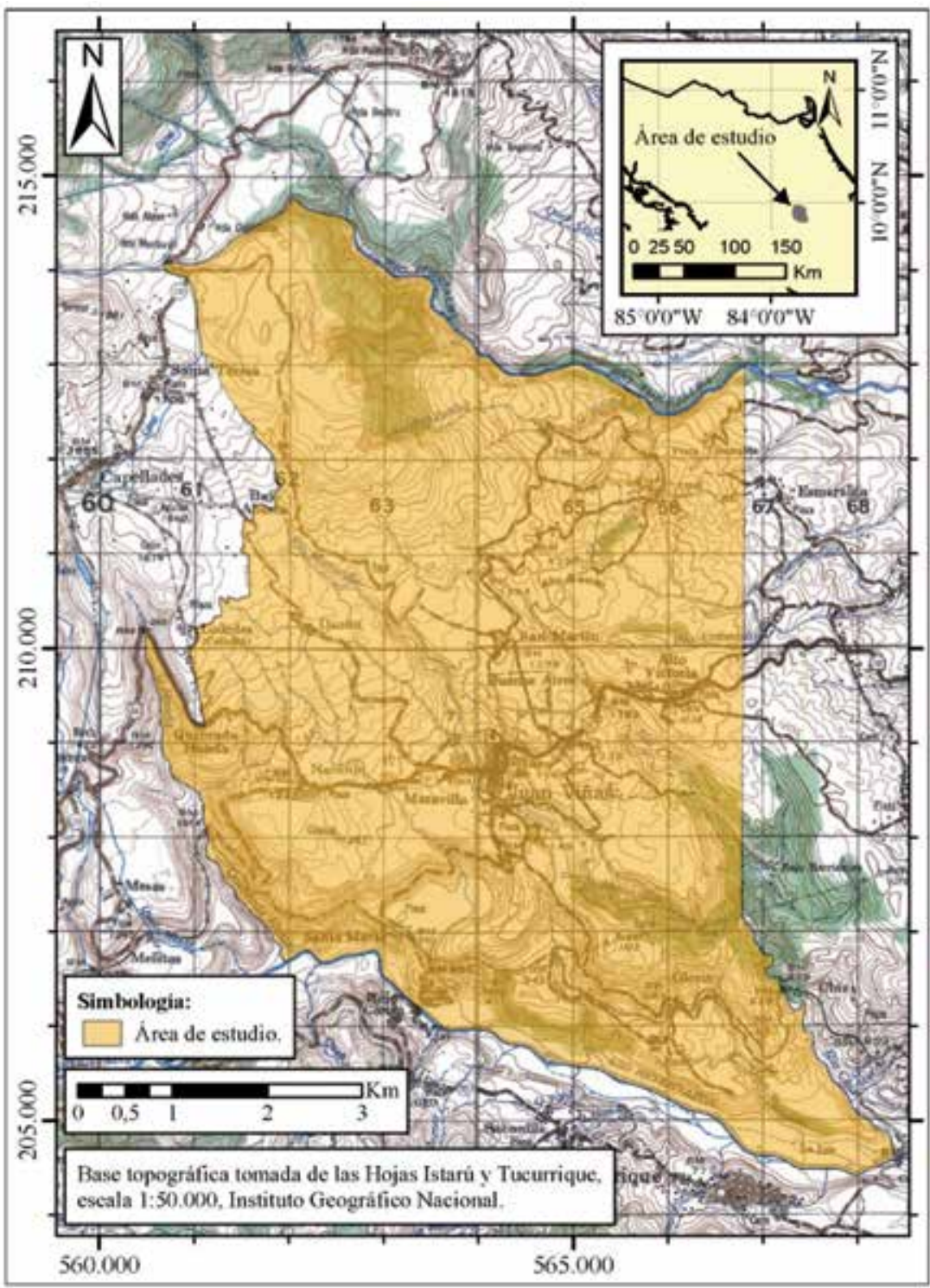

Prácticamente todo el distrito se encuentra dentro de la Hacienda Juan Viñas, la cual utiliza la mayor parte del distrito para producción agrícola. Con respecto a los usos de la tierra, la mayor parte del distrito, del centro del mismo hacia el norte y el sur, concentra su uso de la tierra hacia 
las actividades de siembra de caña de azúcar y en menor medida a cultivos de café. Hacia el norte, cerca del sector de las 50 Manzanas, los usos dominantes corresponden con bosques con algunos pequeños parches de pastos. Hacia el sur por el límite con el río Reventazón los usos de la tierra corresponden en su mayoría a bosques con pequeños parches de charral, pastos y café. La mancha urbana no está del todo desarrollada y se caracteriza por presentarse prácticamente en el centro del distrito y en pequeños caseríos como Esmeralda, San Martín, Naranjo, Santa María y La Gloria. Todo lo anterior corresponde con el trabajo de campo realizado por los autores durante la investigación.

Los ríos principales del distrito son, hacia el norte el río Turrialba, hacia el oeste, la quebrada Honda y al sur el río Reventazón. El río Maravilla y el río Chiz atraviesan el distrito, siendo el primero utilizado para la generación hidroeléctrica privada por parte de la Hacienda Juan Viñas. Hacia el noroeste y oeste, el distrito está influenciado por quebradas efímeras e intermitentes, de las cuales se destacan la quebrada Zetillal, la quebrada Esmeralda y el río Colorado.

\section{Metodología}

Para la delimitación de las unidades geológicas, se realizó una revisión bibliográfica exhaustiva de distintos estudios, Fernández (1987), Mora (1997), Mora (2000), Linkimer (2003), Montero, Rojas \& Linkimer (2013), los cuales constituyen la base geológica regional. Es conveniente mencionar que ninguno de estos estudios constituye un estudio a detalle.

Una vez concluida esta etapa, con la ayuda de mapas topográficos y fotografías aéreas, se realizaron giras de campo para ubicar sitios de afloramientos de materiales litológicos, recolección de muestras macroscópicas para descripción básica y para descripción petrográfica mediante la técnica de sección delgada, este último análisis fue realizado usando los microscopios petrográficos del CICG y ejecutado por los autores. Los sitios de reconocimiento litológico comprendieron no solo los cortes de camino entre los caminos de la finca, sino también en diversas partes del río Maravilla y el río Chiz como también en la carretera hacia el poblado de Tucurrique. La base cartográfica vectorial (curvas de nivel y drenajes) así como las fotografías aéreas utilizadas fueron facilitadas por la Municipalidad de Jiménez. Para construcción del Modelo de Elevación Digital (MED) se utilizó un método 
de interpolación basado en la técnica de interpolación de diferencia finita iterativa utilizando el software Arc Gis 10 debido a que este es correctamente hidrológico y permite representar de manera más apropiado los drenajes lo cual facilita un mayor entendimiento geológico.

Obtenido el MED, se procedió a zonificar el área de estudio partiendo del análisis del MED y del modelo de sombras. Las características de estas unidades brindan una aproximación inicial al origen geológico del distrito.

La corroboración de campo, a partir de descripción de afloramientos, ejecución de ensayos, recolección de muestras macroscópicas para sección delgada, permitió definir y corroborar dichas unidades, de forma tal que se pudiera establecer un primer modelo geológico conceptual del distrito de Juan Viñas. La delimitación de las unidades se basó en las siguientes etapas:

- Delimitación de formas del terreno evidenciadas a partir del patrón de distribución de las curvas de nivel y los patrones de drenajes. Las unidades resultantes se correlacionaron con los procesos geológicos que teóricamente les dieron origen y condicionaron su distribución.

- Mediante el software ArcGis 10, se generó un MED (figura 2) que sirviera de apoyo en la delimitación espacial de las unidades geológicas identificadas. Esta labor incluyó posteriormente el modelado de sombras y la corroboración de unidades en campo. 
Figura 2: Modelo de Elevación Digital del distrito de Juan Viñas, creado a partir de la interpolación de curvas nivel.

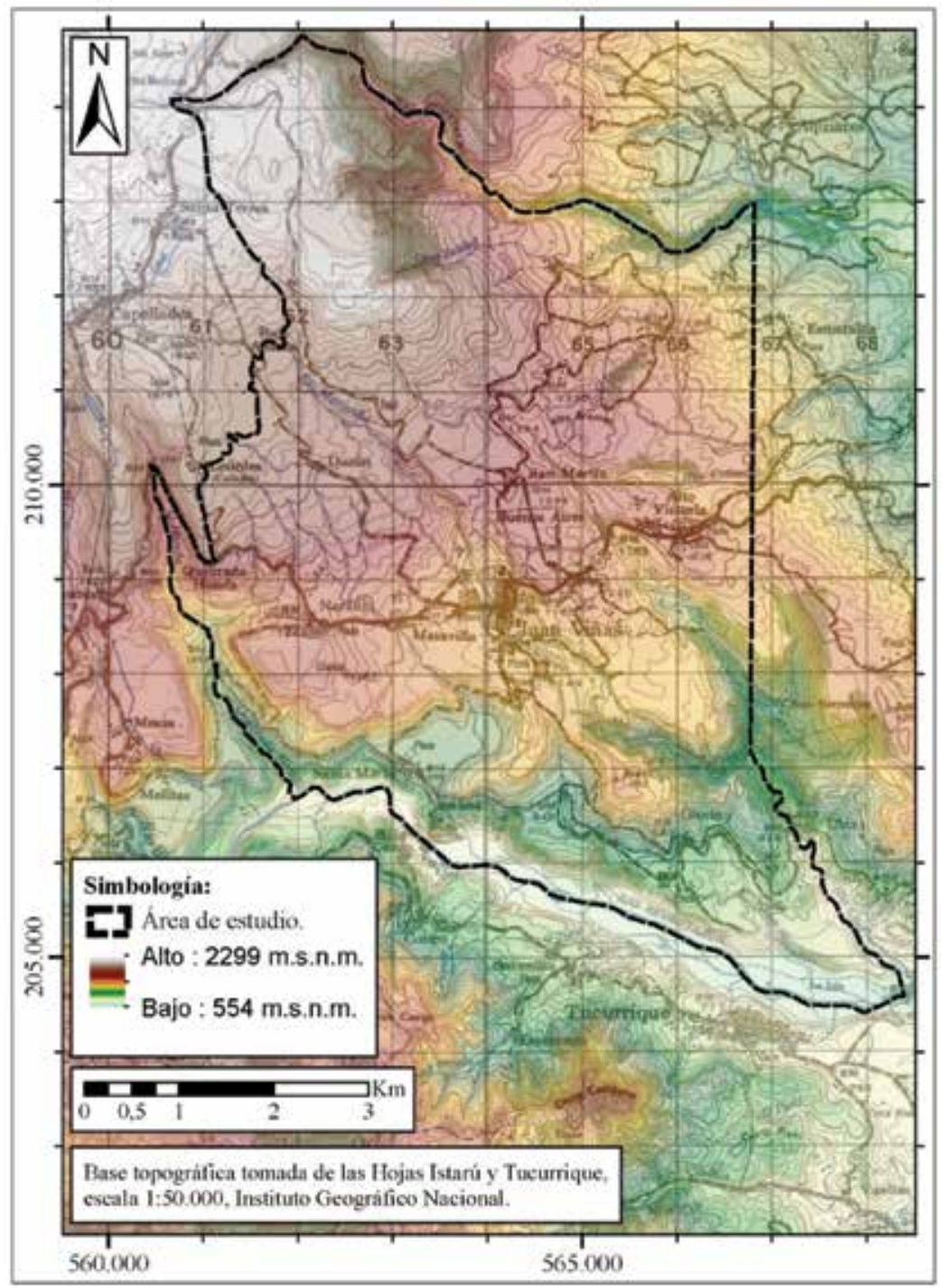

Como se observa en la figura 2, el MED no aporta mayores elementos que permitan la delimitación de las unidades geológicas. Sin embargo, sí es posible sugerir un control estructural sobre los patrones de drenaje (zonas más oscuras de la figura) de los principales ríos y quebradas, los 
Héctor Zúniga Mora, Pablo Ramírez Granados. Setting and characterization of geological units from land numerical model in the district of Juan Viñas, Cartago

cuales se distribuyen en forma paralela a subparalela y orientados generalmente en dirección noroeste - sureste.

Para la construcción de un mejor detalle de la forma del terreno, se utilizó como herramienta el modelado de sombras a partir de las curvas de nivel, que es la técnica que permitió definir las distintas formas del terreno. Así, se delimitaron diez unidades geológicas asociadas con la actividad ígnea (figura 3), así como otras formas originadas por otros procesos geológicos.

Figura 3: Mapa de ubicación del área de estudio.

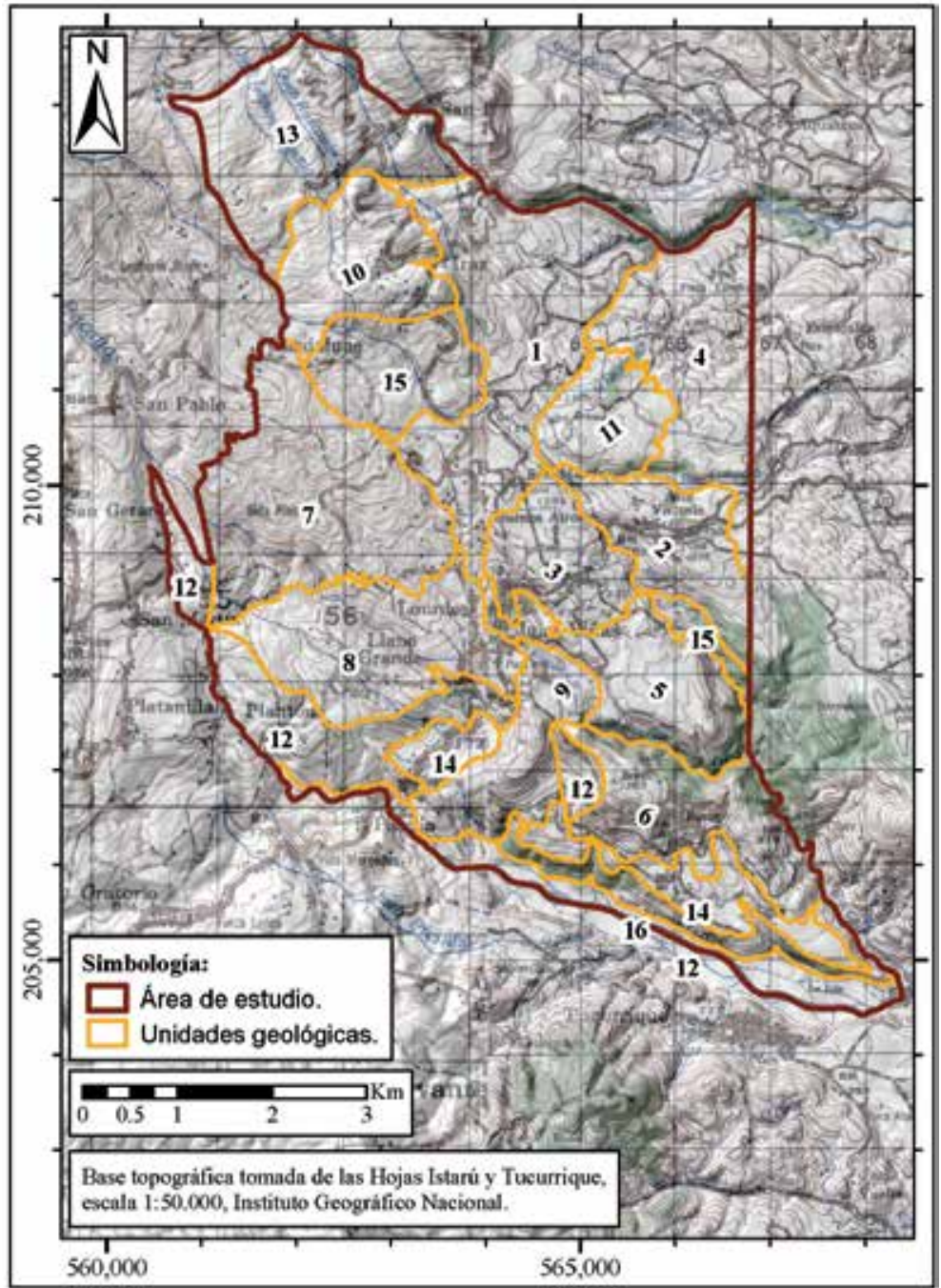




\section{Contexto Geológico Regional}

Fernández (1987) ubica el distrito de Juan Viñas dentro de la Unidad de Lavas, Brecha y Piroclastos de la Formación Andesitas Poás. Este autor indica que predominan las coladas de lava andesítica (augíticas, piroxénicas, olivínicas, hornbléndicas e hipersténicas) y en menor grado se presentan como basaltos olivínicos. En el río Chiz, describe materiales andesíticos hornbléndicos (pardas), con textura hipocristalina porfirítica, matriz intergranular con gran cantidad de material deutérico (Fernández, 1987).

Soto (1988), ubica el área de estudio dentro de lo que denomina el Pre-Turrialba, el cual consta de un edificio volcánico de edad Cuaternaria, conformado por basaltos alcalinos y andesitas ácidas de 2,15 $\pm 0,3$ millones de años. Las estructuras volcánicas presentes en esta unidad tienen una orientación al noreste, al igual que las estructuras volcánicas del volcán Turrialba actual, las cuales se ubican en una zona de fracturas tensionales en la dirección indicada. Kussmaul (2000) indica que la edad de la estructura reciente del volcán Turrialba se estima en 75.000 años.

Soto (1988) sugiere también que estas fracturas pueden deberse a esfuerzos regionales transcurrentes y de bajo corrimiento en la subducción de la Placa del Coco bajo la Placa Caribe.

Más tarde, Mora (1997) propone que el área de estudio se ubica sobre una colada de lava regional llamada "Colada Paraíso", aunque no aborda sus características. No obstante, esta colada corresponde a una delimitación regional realizada a partir de una cartografía a escala 1: 200000.

Geomorfológicamente, Fernández (1987) ubica el área de estudio dentro de la Unidad Colada Aquiares, constituida por lavas andesíticas hasta basálticas y tobas, culminando la secuencia, cuya emisión pudo darse a través de una fractura en las faldas del Volcán Turrialba. Por su morfología, Kussmaul (2000) indica que esta podría ser una de las coladas más jóvenes de la Cordillera Volcánica Central.

En el sector de La Gloria, Fernández (1987) delimitó lo que llamó Paleoterrazas del río Reventazón, específicamente entre los 600 a 700 m sobre el nivel del mar. Se caracterizan por una superficie plano-horizontal muy suavemente inclinada, la cual culmina con escarpes de fuerte pendiente formados por el mismo río. El basamento de las terrazas estaría constituido por rocas volcánicas. 


\section{Unidades Geológicas del Distrito de Juan Viñas}

A partir de la metodología se zonificó el distrito de Juan Viñas en una serie de unidades geológicas las cuales presentan la siguiente descripción:

En el caso de las unidades de origen ígneo, se tratarían de coladas de lava, caracterizadas por estar distribuidas en formas de abanico o completamente concéntricas, dentro de las cuales se han logrado identificar patrones de drenajes radiales típicos de ambientes volcánicos. La distribución de estas unidades se observa en la figura 4 y su descripción geológica es la siguiente:

- Coladas de Lava La Gloria: serían las más antiguas del distrito por estar en el nivel topográfico más bajo y se encuentran ubicadas hacia el sureste. Esta unidad está orientada en dirección oeste-este, y en ella se identifican claramente dos cumbres redondeadas que podrían corresponder con el último punto de emisión de los flujos. Limitan al este con la margen derecha del río Chiz, hacia el oeste con un escarpe de posible origen tectónico, hacia el sur limita con la Unidad Terrazas Aluviales, y hacia el norte con la Unidad Coladas de Lava Juan Viñas. Superficialmente, el perfil de suelo sobre esta colada revela depósitos de lapilli angulares, decimétricos y centimétricos, con bajo contenido de arcilla. 
Figura 4: Unidades geológicas del área de estudio.

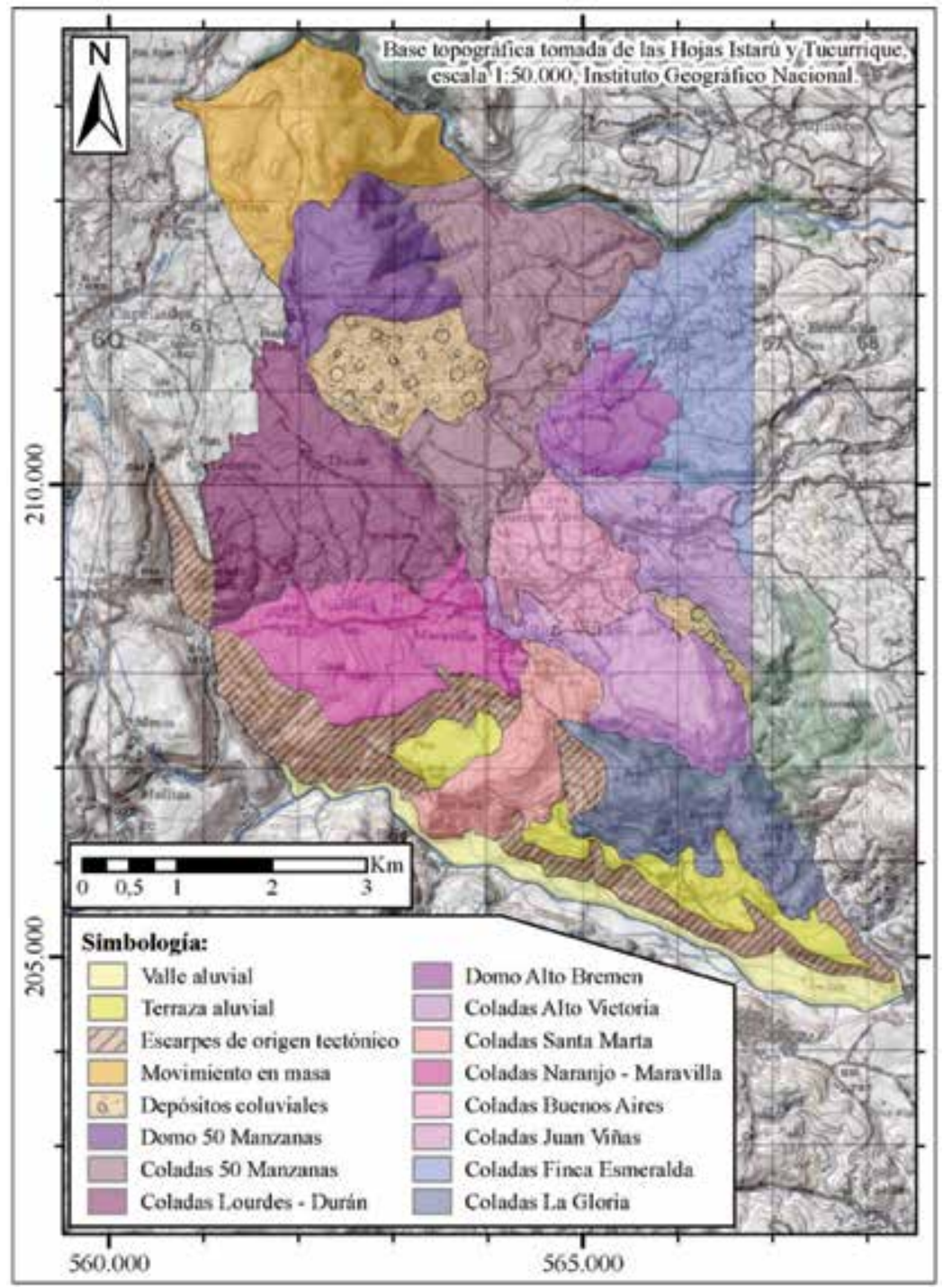

- Coladas de Lava Finca Esmeralda: se ubican hacia el extremo este del área de estudio. El patrón de distribución de las curvas de nivel sugiere que serían flujos de lava originados a partir de varios puntos de emisión, aparentemente alineados en dirección noroeste - sureste. El flujo se habría dado hacia el noreste, hasta la margen derecha 
del río Turrialba. Esta unidad limita al este y al norte con la margen derecha del río Turrialba, hacia el sur y suroeste con la Unidad Coladas de Lava Alto Victoria, y hacia el oeste con el Domo Alto Bremen y con la Unidad Coladas de Lava 50 Manzanas. Localmente, en cortes verticales sobre esta colada, presenta lapilli y toba negra y rojiza de hasta $2 \mathrm{~m}$ de espesor.

- Coladas de Lava Naranjo - Maravilla: se ubican hacia el suroeste del área de estudio. Corresponderían con flujos de lava cuyo último punto de origen se habría ubicado en una loma redondeada hacia el sur de la comunidad de Naranjo. El flujo se habría dado hacia el este, posiblemente llegando más allá de la ubicación actual de la comunidad de Juan Viñas. Esta unidad limita hacia el sur y oeste con un escarpe de origen posiblemente tectónico, hacia el este con las unidades de coladas de lava Santa Marta y Juan Viñas, y hacia el norte con la Unidad Coladas de Lava Lourdes - Durán. Localmente presenta en superficie bloques que van desde los 7 a los $40 \mathrm{~cm}$.

- Coladas de Lava Juan Viñas: se ubican hacia el sureste del área de estudio. Serían flujos de lava en dirección noroeste - sureste, hasta la margen derecha del río Chiz y la margen izquierda del río Maravilla (Juan Viñas); estos dos ríos constituyen el límite sur, este y oeste de esta unidad. El origen de estos flujos no pudo ser inferido a partir del análisis de las curvas de nivel. Estas coladas colindan hacia el norte con la Unidad Coladas de Lava Buenos Aires. Localmente en superficie se manifiestan bloques de tamaño máximo $35 \mathrm{~cm}$ y un promedio de $7 \mathrm{~cm}$.

- Coladas de Lava Santa Marta: su forma claramente alineada en dirección noreste - suroeste, sugiere un origen fisural, por el cual pudo generarse un flujo de alta viscosidad que cubrió parcialmente las coladas Juan Viñas, Naranjo - Maravilla y La Gloria, así como las terrazas aluviales ubicadas al pie de dichas coladas. La distribución de las curvas de nivel sugieren que este flujo alcanzó el valle aluvial actual por el cual fluye el río Reventazón. La sección delgada de esta colada indica una andesita con hornblenda, hipocristalina porfirítica, con presencia de fenocristales de plagioclasa, augita, hornblenda y magnetita envueltos en una matriz hialopilític (Muestra M3 Apéndice A). 
- Coladas de Lava Alto Victoria: esta unidad alineada en dirección noroeste - sureste, sugiere un origen igualmente fisural, por el cual fluyeron coladas en dirección sureste y noreste, cubriendo parcialmente la Unidad Coladas de Lava Finca Esmeralda, con quien aparenta tener un cierto paralelismo.

- Domo Alto Bremen: esta unidad cubriría parcialmente las unidades de coladas de lava Alto Victoria y Fina Esmeralda. Corresponderían con flujos de lava de corta extensión que conforman una estructura subcónica orientada en dirección noreste, donde el último punto de emisión estaría ubicado en la cima del Alto Bremen. Esta cima se ubica sobre el mismo eje en el que se orienta la Unidad Coladas de Lava Alto Victoria, lo que sugiere que ambas unidades pudieron originarse a través de una misma estructura. Aquí el patrón de drenaje muestra una típica distribución radial. En superficie se presentan capas de ceniza de aproximadamente $50 \mathrm{~cm}$.

- Coladas de Lavas Buenos Aires: esta unidad está definida por un frente de colada que se ubica al este y noreste de la comunidad de Juan Viñas, el cual evidencia un flujo que pudo darse en dirección noroeste - sureste. El origen del flujo no pudo ser identificado a partir de las curvas de nivel. Esta unidad colinda al sur con la Unidad Coladas de Lava Juan Viñas, al este con la Unidad Coladas de Lava Alto Victoria, y hacia el oeste y norte con la Unidad Coladas de Lava 50 Manzanas. Aquí el frente de la colada muestra una cresta de forma redondeada y fuerte pendiente.

- Coladas de Lava Lourdes - Durán: corresponde con un frente de colada claramente definido hacia el oeste del área de estudio, el cual evidencia la presencia de coladas que pudieron fluir en dirección noroeste - sureste. El origen de estas coladas no pudo ser determinado a partir de las curvas de nivel, sin embargo, es claro que el mismo podría ubicarse incluso más al noroeste de la comunidad de Capellades (fuera del área de estudio). Esta unidad limita al sur con la Unidad Coladas de Lava Naranjo - Maravilla, al este con la Unidad Coladas de Lava 50 Manzanas, hacia el oeste con un escarpe de origen posiblemente tectónico definido por la margen izquierda de la Quebrada Honda, y hacia el noreste por el Domo 50 Manzanas y por un depósito coluvial al pie de este domo. Superficialmente muestra una capa de 
ceniza meteorizada de aproximadamente $80 \mathrm{~cm}$. Petrográficamente, las rocas de estas coladas se definen como andesita hipocristalina porfirítica, con fenocristales de plagioclasa, augita, hipersteno y magnetita envueltos en una matriz de textura hialopillita con poco vidrio y material criptocristalino (Muestra M9 Apéndice A).

- Conjunto Coladas de Lava y Domo 50 Manzanas: las lavas correspondientes a este conjunto estarían culminando la secuencia en el área estudio. Corresponderían con flujos lávicos hacia el este, sureste y sur, donde la distribución de las curvas de nivel y el patrón de drenaje sugieren que el origen de los mismos estarían bajo el Domo 50 Manzanas, siendo esta última geoforma la que habría culminado la actividad efusiva en la zona. Este domo cuenta con una forma cónica, de base ovalada orienta en dirección noroeste - sureste, el cual muestra en su extremo sureste una estructura de colapso acompañada de un depósito coluvial en su base. Localmente, presenta horizontes limo-arcillosos de $40 \mathrm{~cm}$ de espesor promedio. Superficialmente, las coladas muestran coluvios con bloques centimétricos variando entre los 2 a los $20 \mathrm{~cm}$.

Durante la etapa de reconocimiento de campo, se observa que el techo de estas unidades corresponde generalmente con posibles brechas superiores de colada (autobrechas), las cuales se encuentran muy meteorizadas. Se trata de bloques angulares centimétricos, decimétricos y métricos, de composición homogénea (andesítica generalmente), los cuales se encuentran dentro de una matriz arcillosa de color café - rojizo a naranja. Esta matriz arcillosa podría corresponder con una matriz vítrea completamente arcillificada.

Otros afloramientos permiten observar brechas escoriáceas, menos meteorizadas (relativamente duras); su composición es igualmente andesítica. En estos casos, la mayoría de los cristales de plagioclasa están caolinitizados, o incluso han sido lixiviados, dejando espacios vacíos de forma tabular ("fantasmas") en los espacios donde antes hubo cristales.

Sobreyaciendo estas brechas, se ubica un suelo limo-arcilloso a arcilloso, generalmente muy plástico, de color café - rojizo o café claro, cuyo espesor es de 0,6-1,5 m generalmente. Estas arcillas podrían corresponder con depósitos tobáceos completamente meteorizados. 
En términos generales, esta cobertura de material de caída sobre las brechas sigue una distribución homogénea en lo que respecta a su posible naturaleza, grado de meteorización y distribución, por lo que podría inferirse que estas tobas arcillificadas pudieron ser eyectadas desde un único punto de emisión, cubriendo casi la totalidad del área de estudio.

En el sector de la Unidad de Coladas de Lava Finca Esmeralda, entre los niveles de brechas y tobas arcillificadas color café rojizo, se han detectado también capas intermedias de material igualmente piroclástico. Estas capas corresponden a tobas negras, relativamente sanas, de $0,35 \mathrm{~m}$ de espesor, las cuales muestran un contacto superior transicional de $0,2 \mathrm{~m}$ de espesor, hacia un depósito de lapilli de $0,3 \mathrm{~m}$ de espesor. La secuencia culmina con un depósito de bloques centimétricos de $0,5 \mathrm{~m}$ de espesor.

En la Unidad de Coladas de Lava La Gloria, subyaciendo las tobas arcillificadas café rojizo, se ubican capas alternantes de lapilli y lapilli con arcilla, las cuales, en suma alcanzan espesores que rondan $0,9 \mathrm{~m}$.

Las variaciones en la naturaleza y distribución del material de cobertura registradas en estas dos unidades, sugieren otras fuentes de eyección de piroclastos (posiblemente fuera del área de estudio) cuya influencia fue muy restringida.

Además de las unidades ya descritas, se han delimitado otras cuyo origen se debe a otros procesos geológicos activos, dentro de las que se mencionan:

- Depósitos coluviales: corresponden con superficies irregulares del terreno producto del depósito de material deslizado o removido (generalmente en forma súbita) desde zonas de colapso o desgarre ubicada a mayores altitudes. La mayor extensión correspondiente a esta unidad se ha delimitado al pie del Domo 50 Manzanas, específicamente en su flanco sur. Entre los 30 y $120 \mathrm{~cm}$ en el perfil del suelo, se reconocen bloques de coluvio.

- Movimiento en masa: esta unidad correspondería con un área desestabilizada por un deslizamiento rotacional (slumps), generada por el basculamiento de bloques contra la pendiente sobre una superficie de ruptura circular o semicircular. Esta unidad se caracteriza por presentar superficies onduladas (hummocky), anomalías en la red de drenaje y presencia de zonas pantanosas. La cabecera del deslizamiento estaría al noroeste del área de estudio (fuera de ella), evidenciada por un 
cambio súbito en las pendientes del terreno que define con claridad un escarpe de más de 400 metros de altura, ubicado en el sector de Coliblanco, al noroeste de la comunidad de Santa Teresa de Capellades. Petrográficamente, la muestra recolectada en esta unidad geológica, correspondiente a uno de los bloques movilizados, indica una lava andesita hipocristalina profirítica seriada, con una matriz hialopilitica y fenoscristales de plagioclasa, augita, hornblenda y magnetita (Muestra M4 Apéndice A). Superficialmente de los 0 a los $150 \mathrm{~cm}$ se presentan bloques métricos envueltos en el suelo.

- Escarpes de origen tectónico: corresponde con zonas de muy fuerte pendiente asociadas a fallas geológicas activas presentes en el área de estudio y sus alrededores. Dentro de estas fallas se han identificado las denominadas Capellades y Navarro, propuestas por Montero, Rojas \& Linkimer (2013). Aunque estos escarpes no se ubican exactamente sobre estas estructuras, los mismos pueden corresponder a estructuras paralelas a las mencionadas, o incluso a otras asociadas a estas y que aún no han sido cartografiadas. Las secciones delgadas de las rocas recolectadas en esta unidad geológica, indican que las rocas expuestas en estos escarpes corresponden con andesitas, hipocristalina profirítica, con dominancia de fenocristales de plagioclasa, augita y hornblenda en una matriz hialopilítica. En ambas muestras la matriz es dominante $(60 \%)$ con respecto a los fenocristales $(40 \%)$ con presencia de cristales opacos (Muestras M2 y M1 Apéndice A). Superficialmente debajo de los $200 \mathrm{~cm}$ se presenta coluvio con clastos centimétricos flotando en una matriz limo - arcillosa.

- Terraza aluvial: corresponde con la Unidad Paleoterrazas del Río Reventazón, descrita por Fernández (1987), quien la describe como una superficie plano - horizontal muy suavemente inclinada, la cual culmina con escarpes de fuerte pendiente formados por el mismo río. El basamento de las terrazas estaría constituido por rocas volcánicas, lo cual fue verificado en el campo. Superficialmente bajo los $200 \mathrm{~cm}$ de la superficie, se observan bloques centimétricos en una matriz arcillosa limosa.

- Valle aluvial: corresponde con una zona de baja pendiente sobre la que fluye el río Reventazón. Está constituida por depósitos aluviales generados por la sedimentación de material grueso dentro de una 
matriz generalmente arenosa. Este tramo del río se encuentra aún en la zona de fuerte pendiente de la cuenca, donde los valles no están muy desarrollados y se encuentran restringidos por laderas de fuerte pendiente en ambas márgenes. Superficialmente de los 0 a los 75 $\mathrm{cm}$ de la superficie, se presentan un suelo con materia orgánica y bloques flotantes centimétricos.

\section{Conclusiones}

El distrito de Juan Viñas se ubica sobre flujos de lava regionales, cuyo origen se dio fuera de la misma, esto según varios autores consultados Fernández (1987), Mora (1997), Linkimer (2003), Montero, Rojas \& Linkimer (2013). No obstante, las características de las unidades geológicas descritas, sugieren que la mayoría de ellas pudo generarse dentro del distrito.

La delimitación de las unidades geológicas implicó el modelado del terreno a partir de las curvas de nivel vectoriales proporcionadas por la Municipalidad de Jiménez. Dicho modelado permitió la construcción de un MED a partir del cual se pudo elaborar un mapa de sombras. Este mapa de sombras constituyó la base para la delimitación de las unidades geológicas.

Las unidades autóctonas, lejos de ser parte de los flujos regionales, pudieron ser más bien rodeadas o parcialmente cubiertas por estos, sugiriéndose así un cambio en los conceptos existentes sobre la historia geológica del área de estudio.

La delimitación de unidades geológicas implicó la identificación de los distintos flujos lávicos los cuales facilitan el reconocimiento de unidades de coladas. Adicionalmente a través del trabajo de campo se pudieron delimitar otras unidades originadas por diversos procesos, dichas unidades corresponden con depósitos de materiales, escarpes, terrazas y valles.

Como parte de la identificación específica de las unidades se obtuvieron muestras macroscópicas para su identificación en campo. Aparte de esto se identificó petrográficamente los materiales a partir de secciones delgadas y de muestras granulares procesadas en laboratorio.

Es necesaria la elaboración de más trabajo de campo para la obtención de muestras a profundidad, perforaciones exploratorias y ejecución de ensayos con el propósito de generar un modelo conceptual que constituya una base para procesos de ordenamiento geoterritorial del sitio. 


\section{Referencias}

Denyer, P. \& Alvarado, G. (2007). Mapa geológico de Costa Rica, escala 1:400.000, San José, Librería Francesa.

Fernández, J. (1987). Geología de la hoja topográfica Tucurrique (1:50.000, I.G.N.C.R., $N^{\circ} 3445$ I). (Tesis inédita de licenciatura).

Escuela Centroamericana de Geología de la Universidad de Costa Rica, San José, Costa Rica.

Gutiérrez, M. (2008). Geomorfología. Madrid, España: Pearson Educación, S.A.

Kussmaul, S. (2000). Estratigrafía de las rocas ígneas. En P. Denyer \& S. Kussmaul (Comp.), Geología de Costa Rica, Cartago, Costa Rica: Editorial Tecnológica de Costa Rica, pp.63-86.

Linkimer, L. (2003). Neotectónica del estremo oriental del cinturón deformado del Centro de Costa Rica, (Tesis inédita de licenciatura). Escuela Centroamericana de Geología de la Universidad de Costa Rica, San José, Costa Rica.

Montero, W., Rojas, W. \& Linkimer, L. (2013). Neotectónica de las fallas Ochomogo y Capellades y su relación con el sistema de falla Aguacaliente, falda sur macizo Irazú - Turrialba, Costa Rica. Revista Geológica de América Central, 48, 119 - 139.

Mora, S. (1997). Análisis de la susceptibilidad geológico - geomorfológica a la erosión de la cuenca del río Reventazón, Costa Rica. San José, Costa Rica: Instituto Centroamericano de Administración Pública.

Mora, R. (2000). La variación del nivel freático y su influencia en la capacidad de carga de cimentaciones superficiales. Caso Zapata corrida infinita en un suelo de origen volcánico. Revista Geológica de América Central, 23: 17-23.

Ramírez, P. \& Zúñiga, H. (2014). Estudio hidrogeológico en el distrito de Juan Viñas, Cartago, Informe Final de Investigación, Proyecto 830-B2-039. Centro de Investigaciones en Ciencias Geológicas, Vicerrectoría de Investigación, Universidad de Costa Rica.

Soto, G. J. (1988). Estructuras volcano - tectónicas del Volcán Turrialba, Costa Rica, América Central. V Congreso Geológico Chileno (pp. 163 - 175). Santiago, Chile. 


\section{APÉNDICE A}

Muestra: M3 Roca JV - $03-300512$

Textura: hipocristalina porfirítica seriada un poco glomeroporfirítica con una matriz intersertal.

\section{Fenocristales $40 \%$}

Plagioclasa: idiomiórficas a hipidiomórficas, con tamaño máximo 4 x 1,5 $\mathrm{mm}$, unas muy sanas otras un poco arcillitizadas y corroídas, con textura cribosa, representa un $15 \%$.

Augita: hipidiomórficas a xenomórficas, corroídas, representa un $8 \%$.

Hornblenda: idiomórfica a hipidiomórficas con tamaño máximo de $3 \times 1$ $\mathrm{mm}$, sanas en gran mayoría algunas con bordes de opacita, con colores de interferencia anómalos, representan un $12 \%$.

Magnetita: hipidiomórficas a idiomórficas corroídas, representa un 5\%.

\section{Matriz 60\%}

Matriz con textura hialopilítica con poco vidrio $12 \%$, microlitos de plagioclasa idiomórficas $18 \%$, espacios vacíos $30 \%$.

Nombre: Andesita con hornblenda.

Figura 5: Fotografías de las secciones en Nll izquierda y NX derecha.
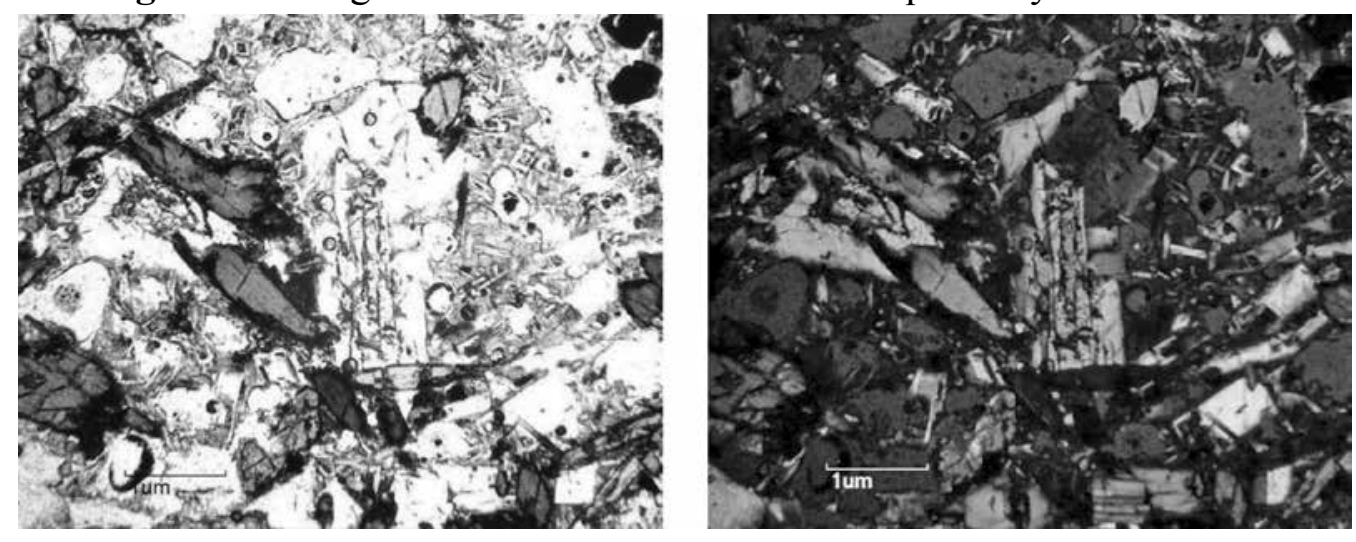
Héctor Zúñiga Mora, Pablo Ramírez Granados. Setting and characterization of geological units from land numerical model in the district of Juan Viñas, Cartago

Muestra: M9 Roca JV - $09-060612$

Textura: hipocristalina porfirítica, con una matriz intersertal, se ve un poco fluidal pues los microlitos de plagioclasa tienen sus ejes largos alineados, además hay óxidos de hierro rellenando algunas vetillas.

\section{Fenocristales $20 \%$}

Plagioclasa: idiomórficas a xenomórficas, arcillitizadas y corroídas algunas son prácticamente fantasmas, con textura cribosa, representa un $12 \%$.

Piroxenos: xenomórficas son fragmentos, representa menos de un $1 \%$.

Opacos: xenomórficas corroídas, algunas tienen un halo amarillo alrededor, representa un $2 \%$.

\section{Matriz 80\%}

Matriz con textura intersertal con poco vidrio menor a $1 \%$, microlitos de plagioclasa hipidiomórficas a xenomórficas alineadas $76 \%$, piroxenos xenomórficos $1 \%$, opacos xenomórficos $2 \%$ y óxidos de hierro $1 \%$.

Nombre: Andesita.

Figura 6: fotografías de las secciones en N1l izquierda y NX derecha
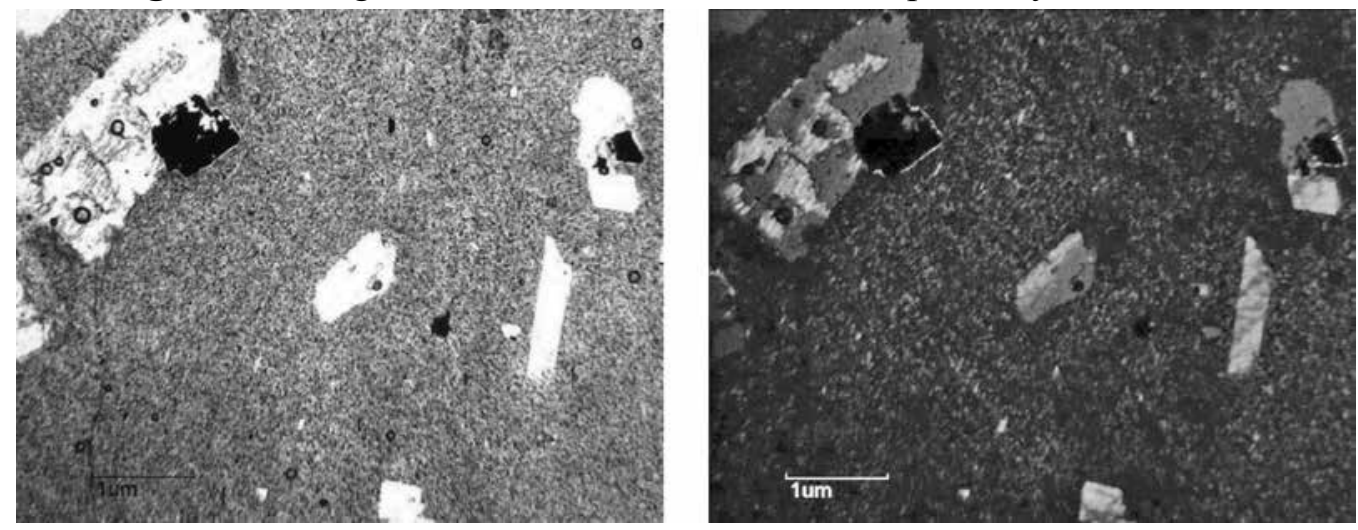
Muestra: M4 Roca JV - $04-06612$

Textura: hipocristalina porfirítica seriada, con una matriz hialopilítica un poco fluidal.

\section{Fenocristales 25\%}

Plagioclasa: idiomiórficas a xenomórficas, con tamaño máximo 5,5 x 1,5 $\mathrm{mm}$, arcillitizadas y corroídas, con textura cribosa, representa un $12 \%$.

Augita: idiomórficas a xenomórficas, corroídas, representa un $8 \%$.

Hornblenda: idiomórfica a hipidiomórfica, algunas con bordes de opacita, representa un $3 \%$.

Magnetita: idiomórfica a xenomórfica, corroída, con tamaño máximo 1 mm aproximadamente, representa un $2 \%$.

\section{Matriz 45\%}

Matriz con textura hialopilítica con vidrio $25 \%$, microlitos de plagioclasa idiomórficas $15 \%$, piroxenos xenomórficos $3 \%$, opacos idiomórficos a xenomórficos $2 \%$.

\section{Nombre: Andesita.}

Figura 7: fotografías de las secciones en N1l izquierda y NX derecha
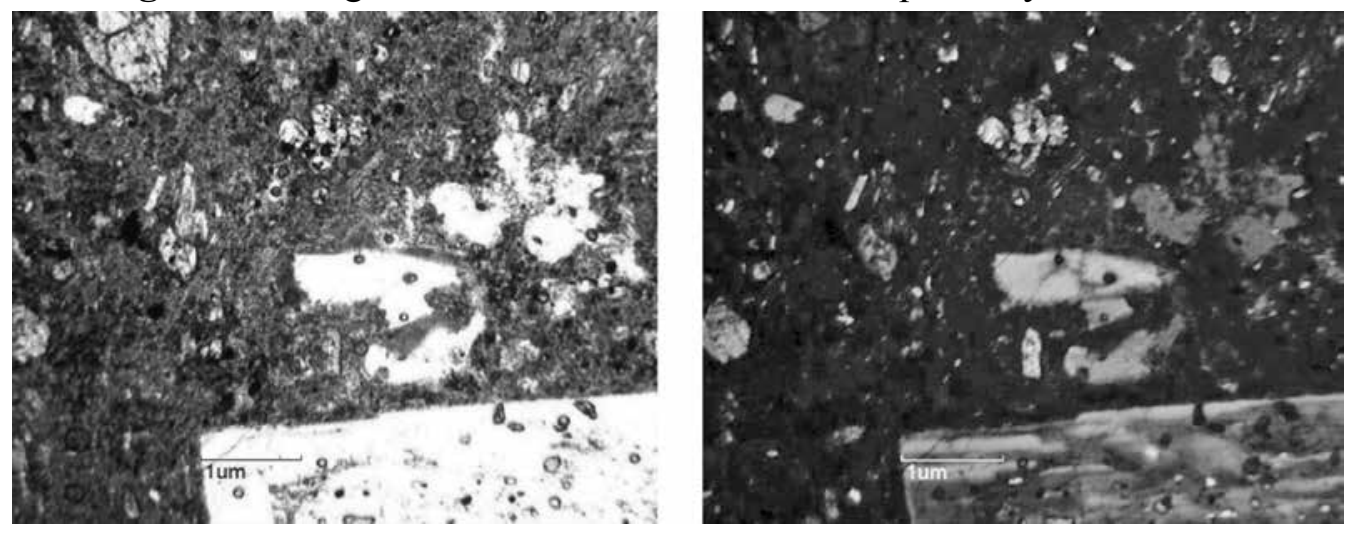
Héctor Zúñiga Mora, Pablo Ramírez Granados. Setting and characterization of geological units from land numerical model in the district of Juan Viñas, Cartago

Muestra: M1 Roca JV - $01-300512$

Textura: hipocristalina porfirítica con una matriz hialopilítica.

\section{Fenocristales $40 \%$}

Plagioclasa: idiomórficas a xenomórficas, arcillitizadas y corroídas con tamaños máximo $6 \times 2 \mathrm{~mm}$, representa un $25 \%$.

Augita: hipidiomórficas a xenomórficas, representa un 10\%.

Opacos: idiomórficas a xenomórficas corroídas, algunas tienen un halo amarillo alrededor, representa un $2 \%$.

\section{Matriz 60\%}

Matriz con textura hialopilítica con vidrio $30 \%$, microlitos de plagioclasa idiomórficas $20 \%$, piroxenos xenomórficos $5 \%$, opacos idiomórficos $5 \%$. Apatito como accesorio.

\section{Nombre: Andesita.}

Figura 8: fotografías de las secciones en Nll izquierda y NX derecha
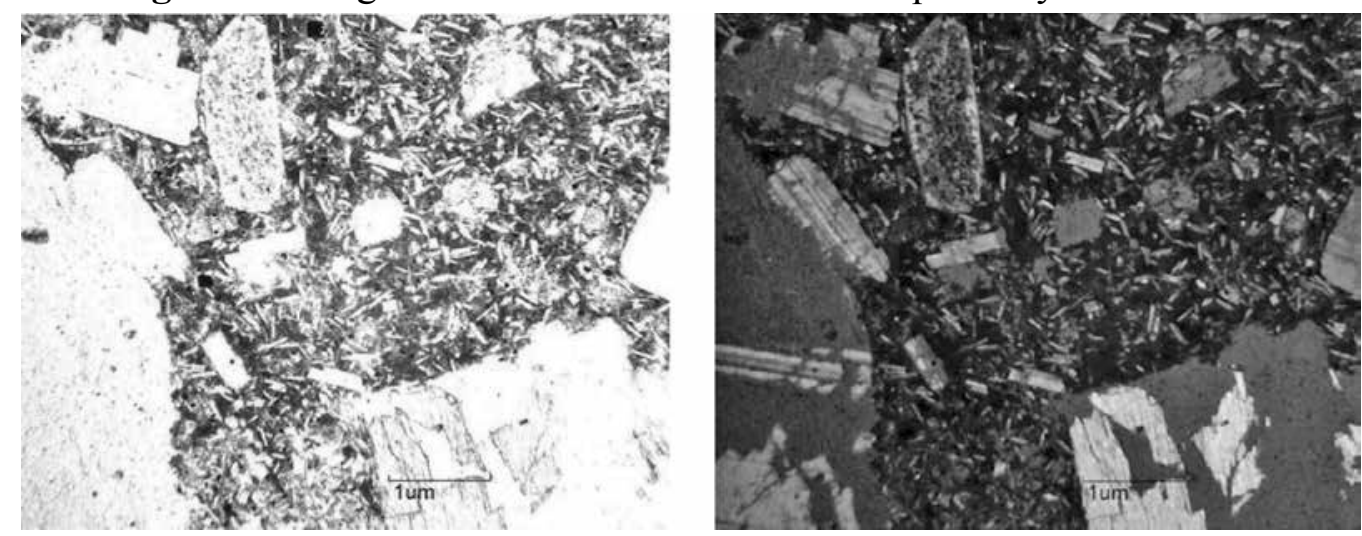
Muestra: M2 Roca JV $-02-300512$

Textura: hipocristalina porfirítica un poco glomeroporfirítica con una matriz hialopilítica.

\section{Fenocristales $40 \%$}

Plagioclasa: idiomiórficas a xenomórficas, arcillitizadas y corroídas, representa un $25 \%$.

Augita: idiomórficas a xenomórficas, hay inclusiones de apatito, representa menos de un $10 \%$.

Hornblenda: cristales idiomórficos, representa un 2\%.

Opacos: xenomórficas corroídas, algunas tienen un halo amarillo alrededor, representa un $2 \%$.

\section{Matriz 60\%}

Matriz con textura hialopilítica con poco vidrio $50 \%$, microlitos de plagioclasa hipidiomórficas a xenomórficas $8 \%$, piroxenos xenomórficos $1 \%$, opacos idiomórficos a xenomórficos $1 \%$.

Apatito como accesorio.

Nombre: Andesita.

Figura 9: fotografías de las secciones en N1l izquierda y NX derecha.
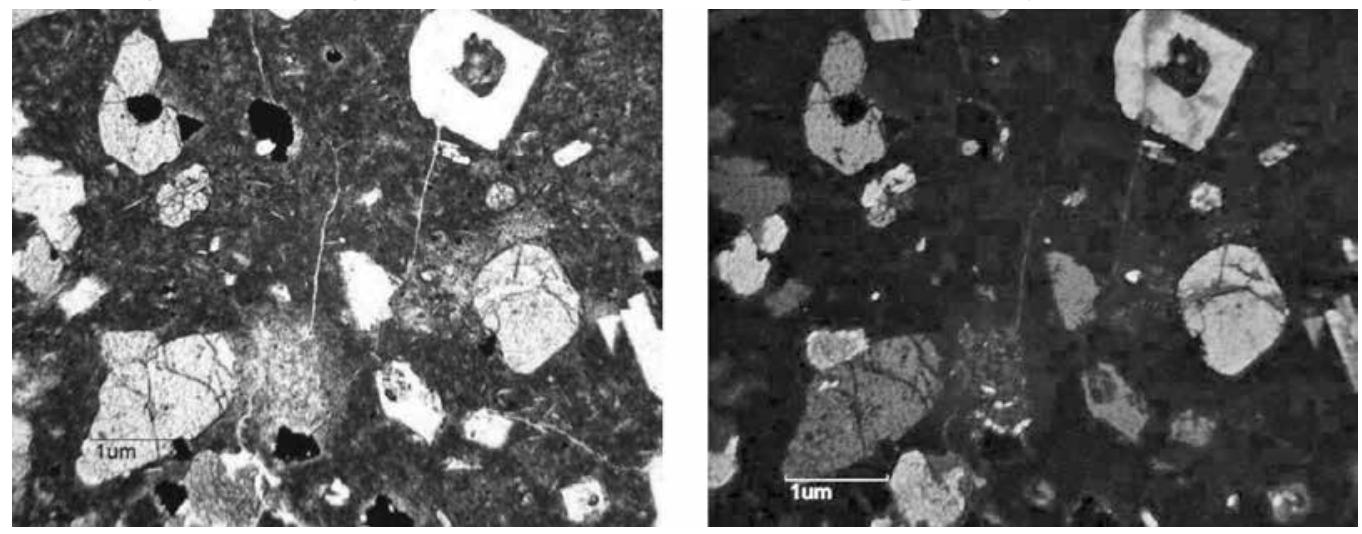